\title{
Mechanical Synthesis of Fullerene-Graphene/Morphed Graphene Architectures
}

\author{
Hector A. Calderon ${ }^{1, *}$, Omar Velazques Meraz ${ }^{2}$ Luis Echegoyen ${ }^{3}$ and Francisco C. Robles Hernandez ${ }^{4, *}$ \\ 1. Instituto Politécnico Nacional, ESFM, Dep. de Física, UPALM, Zacatenco, CDMX 07338 Mexico \\ 2. Centro de Investigacion en Materiales Avanzados (CIMAV), Chihuahua, Chih., Mexico. \\ 3. The University of Texas at El Paso, College of Science, El Paso, Texas 79902, USA \\ ${ }^{4}$ Mechanical Engineering Technology, University of Houston, Houston, TX 77204, USA \\ * Corresponding author: hacalderon@lbl.gov (HAC), fcrobles@uh.edu (FCRH)
}

Experimental evidence of the synthesis of composites of graphene with embedded fullerene molecules is presented. The fullerene is synthesized by conventional electric arc evaporation. The objective of this work is to demonstrate the synthesis of graphene-fullerene and morphed graphene-fullerene architectures where the fullerene is embedded within the graphitic layers. This synthesis is carried out purely by mechanical means using high energy ball milling. First, graphene is produced by milling $0.5 \mathrm{~h}$ and $2 \mathrm{~h}$. Alternatively the synthesis of morphed graphene is conducted by milling carbon soot for longer times, between $10 \mathrm{~h}$ and $30 \mathrm{~h}$. Once the first synthesis is completed it is followed by the synthesis of the composites with the above mentioned architectures. Ideally this is the first step for the development of carbon based superconductors by means of $\mathrm{K}$ doped fullerene. The challenge can be summarized by the differences in sizes between $1.1 \mathrm{~nm}$ [1], $0.33 \mathrm{~nm}$ (d-spacing along "c-axis") [2] and $0.37 \mathrm{~nm}[3,4]$ for fullerene, graphene and morphed graphene respectively. Hitherto we demonstrate that mechanical milling is capable of introducing the fullerene within the graphene layers as well as morphed graphene. The morphed graphenes is more complex than graphene and they are identified as Rh6 and Rh6-II. From a technological viewpoint, the only method to demonstrate this synthesis is by means of low doseatomic resolution TEM because conventional TEM severely damages the material and techniques such as Raman and other methods are incapable of showing changes attributed to this composite.

Figure 1 shows examples of blends of fullerenes and graphenes before the composite is material is actually synthesized. Figure 1a shows a large number of randomly arranged fullernes and graphenes. It is important to notice that the fullerenes and graphenes are only mixed. In Figure $1 \mathrm{~b}$ one can see the fullerene embedded in a rather small graphene particle that is zoomed out and presented in Figure 1c along with the particle size measurements. The abovementioned work was carried out by using a JEOL 200FX and it is clear that a more sophisticated system was needed to identify those particles properly. Thus a Titan and the TEAM 1 microscopes with approximately $55 \mathrm{pm}$ resolution are involved. Figure 2 and 3 conclusively demonstrate not only the presence of fullerene, but how the graphene layers bend in the presence of the embedded fullerene particles. Figure 2 shows a graphene particle and a pristine fullerene in the vicinity to demonstrate the presence of both phases in the blend, but not yet intercalated. The interesting results are those presented in Figure 3 where several cases are shown where fullerene is clearly embedded within the graphene. It is important to notice that graphene is bent along the fullerenes. Furthermore important is the presence of several fullerenes within graphene or single fullerenes intercalated in morphed graphene (Figure 3). Therefore, this clearly demonstrate that this is not an interaction related to the e-beam/particles as this effect is not consistent with the elastic deformation observed in the images. The low dose conditions from the TEAM 1 microscope and the other images in the atomic resolution conditions along with the combination of instruments used herein clearly demonstrate the presence of the molecules embedded in fullerene. For the first time it is shown that fullerene can also be intercalated within morphed graphenes. Conclusively it is shown that the 
complementary combination of microscopes, resolutions and beam conditions are critical to reveal the successful synthesis and existence of the fullerene-graphene and fullerene-morphed graphene architectures. The morphed graphene identified as Rh6-II is purely sp3 bonded and therefore, it has properties similar to those seen in diamond. The fact that we can encapsulate fullerita in morphed graphene may support is the idea that this molecule may be harder than diamond [5].

References

[1] R. Qiao, A.P. Roberts, A.S. Mount, S.J. Klaine, P.C. Ke, Nano Letters 7, 2007, 614.

[2] A.K. Geim, K.S. Novoselov, Nature Materials 6, 2007, 183.

[3] H.A. Calderon, et al., Carbon 102, 2016, 288.

[4] H.A. Calderon, et al., Adv Struct Chem Imaging 2, 2017, 10.

[5] V. Blank, et al., Is C60 fullerite harder than diamond?, Physics Letters A, 188 (1994) 281-286.

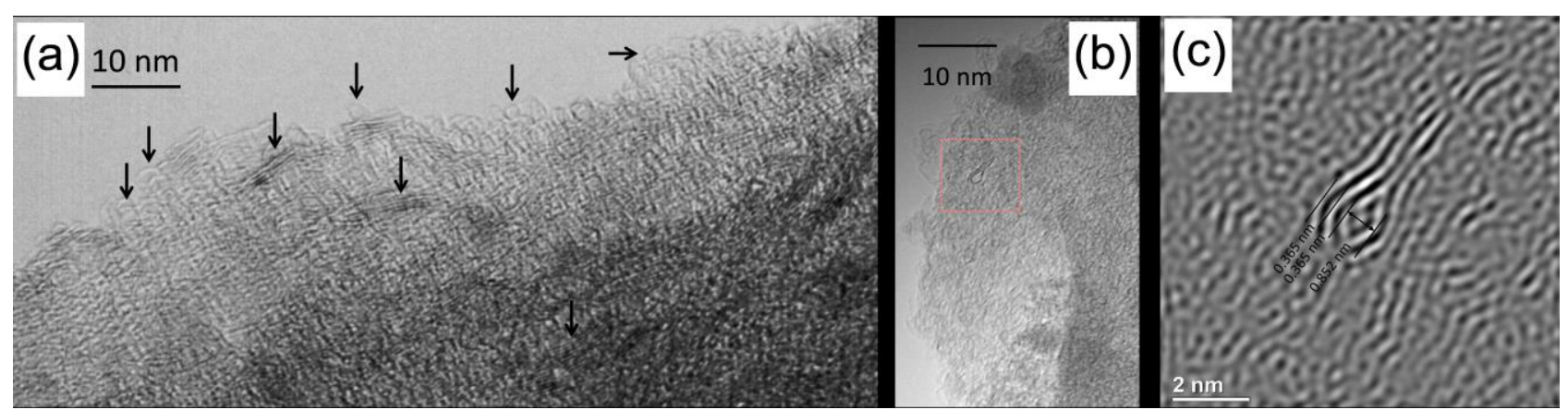

Figure 1. (a) HRTEM images from the blend graphene-fullerene, (b) HRTEM images of a fullerene embedded in graphene and (c) zoom out of (b). The images were made in a JEOL 2000FX with $0.32 \mathrm{~nm}$ resolution.
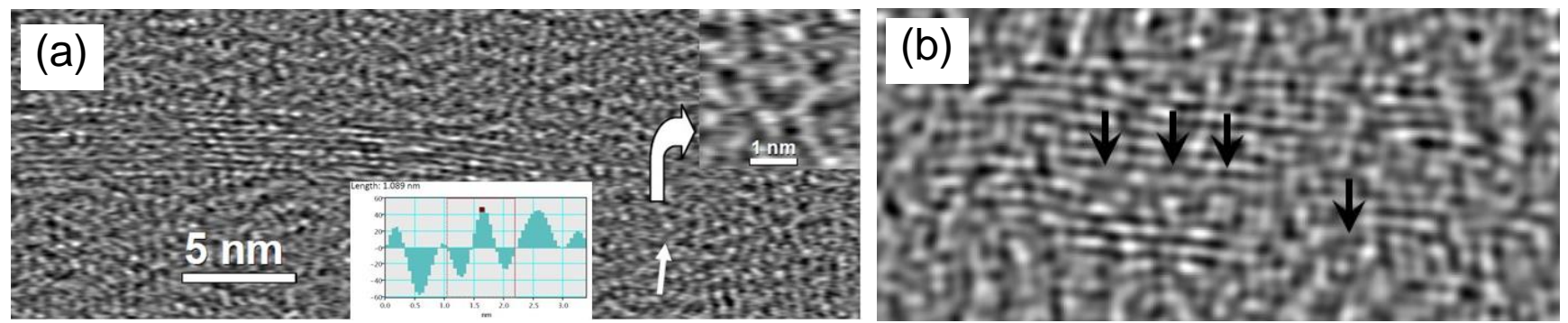

Figure 2. HRTEM (a) showing a fullerene in the vicinity of graphene and (b) four fullerenes within the graphene. The length of the fullerene was measured to demonstrate its size. The images from a Titan operated at $300 \mathrm{kV}$ with $\sim 55 \mathrm{pm}$ resolution.
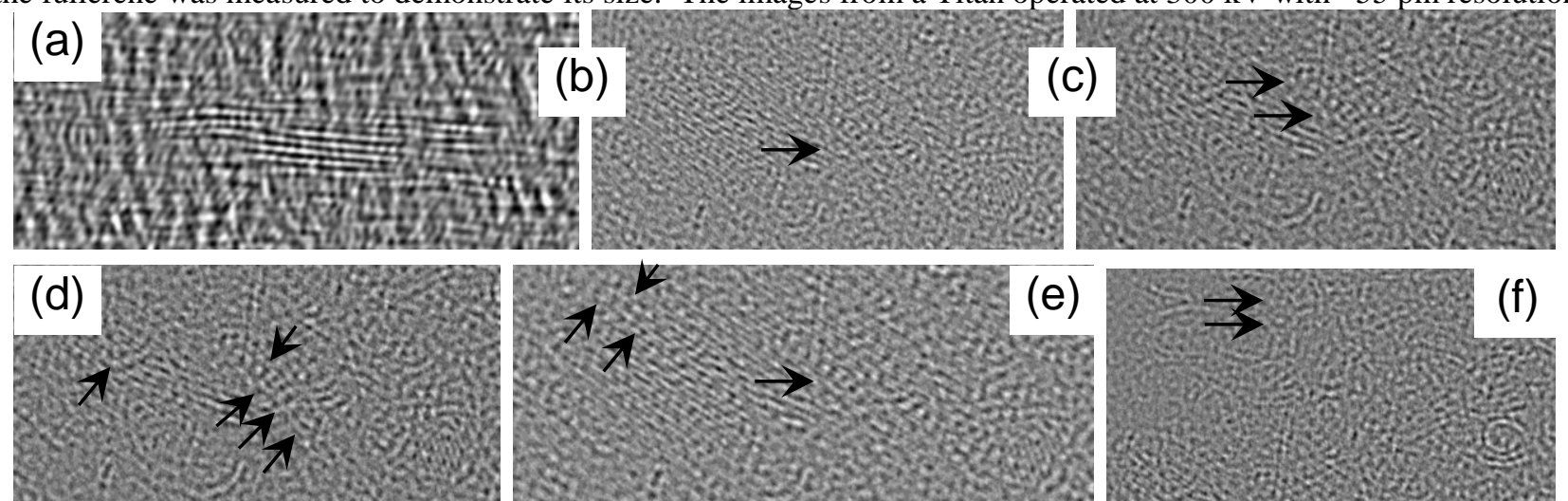

Figure 3. (a) Bent graphene along the fullerene molecule and (b-f) focal series showing a number of fullerenes within the graphene and morphed graphene areas. The images were made in a (a) Titan operated at $300 \mathrm{kV}$ and (b-f) TEAM 1 operated at $80 \mathrm{kV}$ under low dose conditions and the images were collected using a Gatan K2 camera. 